\title{
Epidemic Dynamics in Open Quantum Spin Systems
}

\author{
Carlos Pérez-Espigares, ${ }^{1}$ Matteo Marcuzzi, ${ }^{1}$ Ricardo Gutiérrez, ${ }^{1,2}$ and Igor Lesanovsky ${ }^{1}$ \\ ${ }^{1}$ School of Physics and Astronomy, University of Nottingham, Nottingham NG7 2RD, United Kingdom \\ and Centre for the Mathematics and Theoretical Physics of Quantum Non-equilibrium Systems, \\ University of Nottingham, Nottingham NG7 2RD, United Kingdom \\ ${ }^{2}$ Complex Systems Group, Universidad Rey Juan Carlos, 28933 Móstoles, Madrid, Spain.
} (Received 6 June 2017; published 4 October 2017)

\begin{abstract}
We explore the nonequilibrium evolution and stationary states of an open many-body system that displays epidemic spreading dynamics in a classical and a quantum regime. Our study is motivated by recent experiments conducted in strongly interacting gases of highly excited Rydberg atoms where the facilitated excitation of Rydberg states competes with radiative decay. These systems approximately implement open quantum versions of models for population dynamics or disease spreading where species can be in a healthy, infected or immune state. We show that in a two-dimensional lattice, depending on the dominance of either classical or quantum effects, the system may display a different kind of nonequilibrium phase transition. We moreover discuss the observability of our findings in laser driven Rydberg gases with particular focus on the role of long-range interactions.
\end{abstract}

DOI: 10.1103/PhysRevLett.119.140401

Introduction.-Cold atoms and ions are versatile platforms for the exploration of nonequilibrium physics. Recent examples include studies on creation and dynamics of quasiparticles [1,2], spreading of entanglement and correlations [3-5], as well as many-body localization in disordered systems [6-8]. In particular, so-called Rydberg gases with their strong long-range interactions $[9,10]$ permit the exploration of open and closed many-body physics [11-15], with recent experiments probing nonequilibrium dynamics [15-18], phase transitions [19-22], and disorder-induced localization phenomena [23].

An intriguing aspect is that in Rydberg gases one can achieve control over the relative strength of quantum fluctuations and classical noise [10]. This permits the exploration of dynamical phenomena in settings that can be regarded as quantum generalizations of classical nonequilibrium systems $[24,25]$. A recent example is a quantum version of the so-called contact process [26,27], a simple stochastic model for population dynamics featuring a nonequilibrium phase transition [28] whose character changes drastically when moving from a purely classical to a quantum regime.

In this work we shed light on the collective dynamics of an open quantum system generalization of a general epidemic process (GEP) [29], belonging to the dynamic percolation universality class [28,30-32]. In a Rydberg system, a similar dynamics can be expected by considering atoms with three relevant states, which can be labeled as "healthy," "infected," and "immune," where infected sites have the ability to infect their healthy neighbors, or heal and become immune. A scenario similar to this has been recently realized and studied experimentally in Ref. [15], where a connection to the GEP was conjectured. The scope of this work is not to propose a quantum simulation protocol for GEP, but to demonstrate that, under dominant classical noise, the system follows the same phenomenology as the GEP and undergoes a continuous transition between two phases: one where the contagion starting from an initially infected site is unable to percolate throughout the system, and one where the initial infected site triggers a self-sustaining wave front (an "outbreak") which covers an extensive portion of the system and leaves behind a trail of immunized sites. In the quantum regime, a mean-field treatment suggests that the density of immune sites displays a sequence of jumps resulting from the presence of multiple wave fronts.

Model.-We consider atoms with three internal states: a ground state $|g\rangle$ (healthy), a Rydberg state $|r\rangle$ (infected), and a second stable state $|n\rangle$ (immune). These states are coupled as depicted in Fig. 1(a): $|g\rangle$ is excited to $|r\rangle$ via a laser with Rabi frequency $\Omega$ and detuning $\Delta$ and the state $|r\rangle$ decays radiatively into $|n\rangle$ at rate $\kappa$. Note that this implicitly assumes that the decay from $|r\rangle$ does not proceed via long-lived intermediate states. Radiative decay from $|r\rangle$ to $|g\rangle$ is neglected for simplicity (see Ref. [33] for details). The atoms are placed on a two-dimensional square lattice with $L$ sites and spacing $a$, one per site (see, e.g., experiments in Refs. [38,39]).

Collective behavior emerges when the probability for an atom to undergo the transition $|g\rangle \rightarrow|r\rangle$ (infection of a healthy site) depends on the state of its neighbors. For Rydberg atoms this is achieved by enforcing the so-called "facilitation" (or "antiblockade") condition [13,16,40-44]. Here, the detuning $\Delta$ of the excitation laser is set to compensate the interaction $V_{\mathrm{NN}}$ between neighboring atoms, which makes the transition $|g\rangle \rightarrow|r\rangle$ resonant provided a neighbor is already in state $|r\rangle$ [Fig. 1(a)]. This situation has already been explored in a two-level 


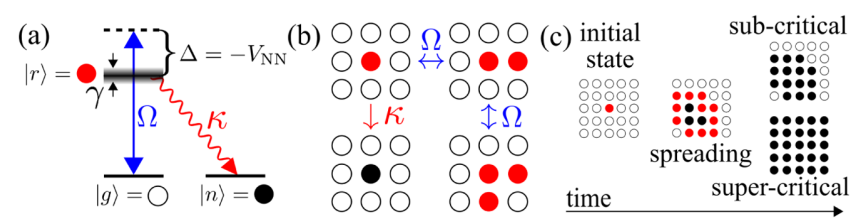

FIG. 1. (a) Atoms on a square lattice are coherently excited from the ground state $|g\rangle$ to a Rydberg state $|r\rangle$ with a laser with Rabi frequency $\Omega$. External noise broadens the state $|r\rangle$ (width $\gamma$ ) which decays to a third state $|n\rangle$ at rate $\kappa$. The laser is off resonant with a detuning $\Delta$ that compensates the nearest-neighbor interaction $V_{\mathrm{NN}}\left(V_{\mathrm{NN}}-\Delta=0\right)$. (b) The dominant processes are facilitation (top row and right column) and decay (left column). (c) An initial seed leads to the formation of clusters of Rydberg states (infected sites) which can either be converted to ground state atoms (healthy sites) or decay to the immune state $|n\rangle$. The relative strength of the dephasing rate $\gamma$ with respect to $\Omega$ determines the nature of the transition. At fixed $\gamma$, depending on the ratio $\Omega / \kappa$ the stationary state is either an ever-expanding infection leaving a macroscopic fraction of immune sites (supercritical) or an infection that dies and leaves a lattice partially (not macroscopically) filled (subcritical).

setup $[12,13,16,45]$, and very recently also in the considered three-state setting [15]. We remark that it is crucial for infection to only occur locally, i.e., in the neighborhood of an already infected site. In our case, it is therefore important that the interactions decay sufficiently fast with the distance. A variety of different behaviors are known to emerge in systems where they do not [46-49].

We now consider a minimal model of the resulting many-body dynamics in which the density matrix $\rho$ of the system evolves under a Lindblad master equation [50] $\partial_{t} \rho=-i[H, \rho]+\sum_{k=1}^{L}\left[\kappa \mathcal{L}\left(|n\rangle_{k}\langle r|\right) \rho+\gamma \mathcal{L}\left(r_{k}\right) \rho\right]$, whose terms are sketched in Fig. 1(b). The coherent evolution is governed by the Hamiltonian $H=\Omega \sum_{k} \Pi_{k} \sigma_{k}^{x}$, where $\sigma_{k}^{x}=|g\rangle_{k}\langle r|+| r\rangle_{k}\langle g|=\sigma_{k}^{-}+\sigma_{k}^{+}$, and $\Pi_{k}$ is a projector onto the subspace in which exactly one of the nearest neighbors of $k$ is in state $|r\rangle$. Denoting the set of nearest neighbors of $k$ by $\Lambda_{k}$, it reads

$$
\Pi_{k}=\sum_{l \in \Lambda_{k}} r_{l} \prod_{m \in \Lambda_{k} \backslash\{l\}}\left(1-r_{m}\right)=\sum_{l \in \Lambda_{k}} r_{l}+\ldots,
$$

with $r_{k}=|r\rangle_{k}\left\langle r\left|, g_{k}=\right| g\right\rangle_{k}\langle g|$ and $n_{k}=|n\rangle_{k}\langle n|$. The dots denote higher-order terms in the operators $r_{k}$. This projector constrains infection to occur only in the neighborhood of a single infected site. The dissipative dynamics is described by Lindblad terms $\mathcal{L}(J) \rho=J \rho J^{\dagger}-\left\{J^{\dagger} J, \rho\right\} / 2$. The first describes decay from $|r\rangle$ to $|n\rangle$ at rate $\kappa$, the second dephasing of quantum coherences at rate $\gamma$. Controlling the dephasing strength, which is achievable by modifying the excitation laser linewidth or the temperature of the atoms, allows switching between classical and quantum regimes [10,51]. In the following, the initial state is always a single atom in state $|r\rangle$ (infected) in the center of the lattice and all the others in state $|g\rangle$ (healthy).
Classical regime.-We first consider the regime of strong dephasing $\gamma \gg \Omega$. Here an effective dynamics can be defined for the diagonal of the density matrix $\mu_{i j}=\delta_{i j} \rho_{i i}$ in the $|r, g, n\rangle$ basis and the corresponding (classical) master equation reads [52,53]

$\partial_{t} \mu=\sum_{k}\left[\alpha \Pi_{k}\left(\mathcal{L}\left(\sigma_{k}^{+}\right)+\mathcal{L}\left(\sigma_{k}^{-}\right)\right)+\kappa \mathcal{L}\left(|n\rangle_{k}\langle r|\right)\right] \mu$,

with $\alpha=4 \Omega^{2} / \gamma$. This means that atoms undergo incoherent state changes from $|g\rangle$ to $|r\rangle$ and vice versa, with a rate conditioned on their local neighborhood. Furthermore, decay from $|r\rangle$ to $|n\rangle$ is possible. The process (2) is similar to a GEP but differs from it by (i) the presence of facilitated transitions $|r\rangle \rightarrow|g\rangle$ and (ii) for the stricter constraint that a single neighboring infected site is required for facilitation, whereas in the GEP the infection rate is proportional to the number of infected neighbors.

In a GEP, for fixed $\kappa$, if the facilitation rate lies below its critical value, the initial infection is unable to propagate and the density of immunes $N=\sum_{k}\left\langle n_{k}\right\rangle / L$ ( $L$ being the number of sites) vanishes in the thermodynamic limit. Conversely, above the critical point there is a finite probability for the infection to percolate [see Fig. 1(c)], propagating as a single traveling wave front and leaving behind a finite fraction of immune sites $N>0$ [29] (i.e., a single outbreak takes place).

We start from a uniform mean-field approximation where we neglect the higher order terms in Eq. (1), effectively relaxing (ii). Introducing the quantities $R=$ $\sum_{k}\left\langle r_{k}\right\rangle / L$ and $G=\sum_{k}\left\langle g_{k}\right\rangle / L$ yields

$$
\begin{aligned}
& \partial_{t} G=-4 \alpha R(G-R), \quad \partial_{t} N=\kappa R, \\
& \partial_{t} R=4 \alpha R(G-R)-\kappa R .
\end{aligned}
$$

Analogously to what is found for the GEP [29], these equations feature a constant of motion $\partial_{t}[\log (R-G+\kappa / 8 \alpha)+$ $8 N \alpha / \kappa]=0$, which permits the determination of the stationary phase diagram for different initial conditions shown in Fig. 2(a) [33]. In a uniform approximation, the closest initial condition to the one we start from is a vanishingly small density of infections $\left[R(t=0)=\epsilon \rightarrow 0^{+}\right]$and in this limit the two phases [which are illustrated in Fig. 1(c)] can be clearly identified, separated by a critical point at $\alpha_{c}=\kappa / 4$. The nonuniform mean-field dynamics is shown in Fig. 2(b) and highlights the absence or presence of an outbreak in the two phases.

We have then performed continuous-time Monte Carlo simulations of the classical master equation (2). In Fig. 2(c) we show the stationary density $N_{\mathrm{SS}}=$ $\lim _{t \rightarrow \infty} N(t)$ for different system sizes and observe a sharpening crossover from a vanishing to a finite-valued phase when increasing $L$. The dynamics shows the expected GEP behavior: for $\alpha<\alpha_{c} \approx 1.71 \kappa$ the process fails to percolate and no outbreak is produced. In the 


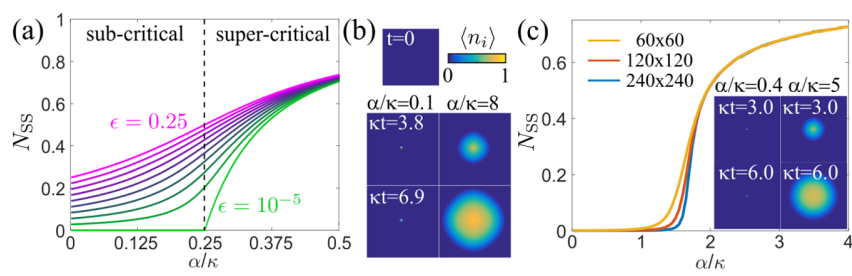

FIG. 2. (a) Stationary state of the mean-field equations (3). Numerical solution after time $\kappa t=500$ for different initial conditions: $R(t=0)=\epsilon, G(t=0)=1-\epsilon$, and $N(t=0)=0$. (b) Evolution of $\left\langle n_{i}\right\rangle$ on a $L=51 \times 51$ lattice starting at $t=0$ from a single site in state $|r\rangle$ located at the center of the lattice. In the supercritical regime the epidemics spreads through the lattice leaving a region of immune sites behind, while in the subcritical regime the spreading soon halts. (c) The Monte Carlo simulation of (2) displays a continuous phase transition as well, with order parameter profiles becoming sharper the larger the system size. Snapshots are averaged over $10^{4}$ realizations and display the expected qualitative features.

supercritical phase, instead, there is a finite probability of a single outbreak immunizing a macroscopic portion of the system. A finite-size scaling analysis performed with the tabulated critical exponents for the GEP provides an excellent collapse of the curves [33], establishing the connection on firm grounds. The full constraint given by Eq. (1) leads to the same scaling behavior near the critical point. This could be expected since the differences between our process and the GEP are of higher order in the density of infected sites, which vanishes close to the critical point. More rigorously, one can show that these terms are irrelevant in a renormalization-group sense [33].

Rydberg gases.-We now discuss the observability of this physics in Rydberg gases interacting with a power-law potential $V(r)=C_{\beta} / r^{\beta}$ (here we consider the van der Waals case $\beta=6$ ) under antiblockade conditions. As shown in Refs. [44,53-55], in this case the constraint $\Pi_{k}$ is replaced by the operator $\Gamma_{k}=$ $1 /\left\{1+R^{2 \beta}\left[1-\sum_{m \neq k}\left(r_{m} /\left|\mathbf{x}_{k}-\mathbf{x}_{m}\right|^{\beta}\right)\right]^{2}\right\}$, where $R=$ $\left(2 C_{\beta} / a^{\beta} \gamma\right)^{1 / \beta}$ is the so-called dissipative blockade radius and $\mathbf{x}_{k}$ denotes the position of the $k$ th atom. The lattice spacing $a$ is taken as the facilitation distance: if just one nearest neighbor of site $k$ is infected, the rate is maximized, i.e., $\Gamma_{k}=1$, whereas other configurations lead to its suppression. In this context, two main effects alter the physics of the previous model: the possibility of unfacilitated infection $(|g\rangle \leftrightarrow|r\rangle$ in the absence of infected neighbors) and the fact that the interactions extend beyond nearest neighbors [21]. To gain insight into their role, we exploit the rapid decay of the tails of $V(r)$ to truncate the interaction beyond a distance of two lattice sites,

$\Gamma_{k}^{-1} \approx 1+R^{2 \beta}\left(1-\sum_{l \in \Lambda_{k}} r_{l}-\eta \sum_{m}^{\prime} \frac{r_{m}}{\left|\mathbf{x}_{k}-\mathbf{x}_{m}\right|^{\beta}}\right)^{2}$, where the primed sum runs only over $m$ such that $1<\left|\mathbf{x}_{k}-\mathbf{x}_{m}\right| \leq 2$. Here we have introduced a parameter $\eta$, which allows us to control the strength of the "longrange" part. While this parametrization is used here for convenience, in practice potential shaping techniques can be applied to modify and possibly suppress the potential tails; see, e.g., Refs. [55-57].

First, we consider $\eta=0$ (nearest-neighbors interactions only), where $\Gamma_{k}$ is well approximated by the constraint $\Pi_{k}$ [Eq. (1)], provided that $R>1$. Unfacilitated (spontaneous) infection can now occur at a rate $\alpha \Gamma_{\text {spont }}=\alpha /\left(1+R^{2 \beta}\right)>0$. Albeit rare for $R \gg 1$, these processes dramatically alter the stationary-state properties of the system, invariably leading to $N_{\mathrm{SS}}=1$. In the renormalization-group language, the spontaneous processes constitute a relevant perturbation. Nevertheless, for sufficiently large $R$ a time scale separation occurs: the outbreak follows the phenomenology observed for the idealized case up to times on the order of $\kappa t \sim\left(1+R^{2 \beta}\right) /(L \alpha / \kappa)$, which is an underestimate of the mean waiting time of the Poisson process producing spontaneous infection. This is illustrated in Fig. 3(a), where we show the stationary density of immune states $N_{\mathrm{SS}}$ of the idealized process (rate function $\alpha \Pi_{k}$ ), together with the density $N(t)$ at time $\kappa t=100$ for the Rydberg rates with $R=5, \beta=6$, and $\eta=0$. These curves display remarkable agreement showing that this phase transition in fact underlies the transient Rydberg dynamics. In the inset of Fig. 3(a) we moreover show how the characteristic sigmoidal profile remains stable for a long period of time. In Fig. 3(b) we show that outbreaks are visible for some time, but spontaneous infection leads to a rising "background" that

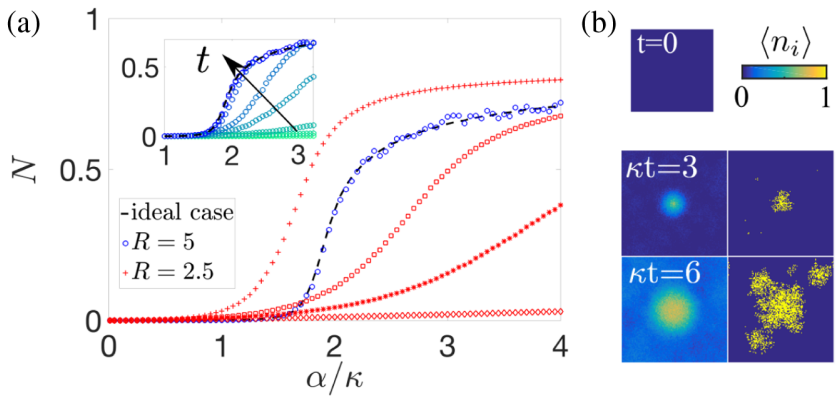

FIG. 3. (a) Mean density of immune states in the ideal case with rates $\alpha \Pi_{k}$ (1) (black dashed line), and in the Rydberg case (4) at time $\kappa t=100$ for $R=5$ and $\eta=0$ (blue circles) and for $R=2.5$ and different values of $\eta=0,0.05,0.1,1$ (red symbols from top to bottom). The Rydberg model prediction matches the idealized model's when the effects of the potential tails and of spontaneous excitation can be neglected. Inset: Mean density of immune states using the Rydberg constraint (4) with $R=5, \beta=6$, and $\eta=0$ for increasing values of time $\kappa t=3,6,10,20,30,60,90$, and 100. The ideal case with constraints $\Pi_{k}$ (black dashed line) is again included for comparison. (b) Evolution of the density of immune states averaged over 100 realizations (left column) and for a single realization (right column) on a $L=120 \times 120$ lattice for $\alpha=5 \kappa, R=2.5$, and $\eta=0$ at two different values of $\kappa t$. 
eventually overcomes the epidemic process and immunizes the entire system.

Long-range interactions $(\eta>0)$ counteract the formation of large clusters of infected and immune sites. For instance, an isolated atom in state $|r\rangle$ can infect, say, the site directly below it at the maximal rate, since the nearest-neighbor interaction compensates the laser detuning. To infect a third one at the right of the former, however, one has to include the additional shift due to next-nearest-neighbor interactions, which brings the atomic transition off resonance, hindering the propagation. We illustrate this by showing in Fig. 3(a) the density of immune states at time $\kappa t=100$ for $R=2.5$ and different values of $\eta$ (all red symbols). As $\eta$ is increased, it becomes more difficult for the process to spread. This, however, is a lattice effect; in dense atomic clouds the transition may reappear. This is suggested by recent experimental works $[13,15,16,21]$ that reveal collective phenomena in the presence of thermal motion.

Quantum regime.-We now set the dephasing rate $\gamma=0$ and study what we refer to as the "quantum case." We describe the dynamics via the mean-field equations

$\partial_{t} G_{k}=-\Omega \bar{R}_{k} \Sigma_{k}, \quad \partial_{t} R_{k}=\Omega \bar{R}_{k} \Sigma_{k}-\kappa R_{k}$,

$\partial_{t} N_{k}=\kappa R_{k}, \quad \partial_{t} \Sigma_{k}=-2 \Omega \bar{R}_{k}\left(R_{k}-G_{k}\right)-\frac{\kappa}{2} \Sigma_{k}$.

Here, $R_{k}\left(G_{k}, N_{k}\right)=\left\langle r_{k}\left(g_{k}, n_{k}\right)\right\rangle$ and $\Sigma_{k}=\left\langle\sigma_{k}^{y}\right\rangle$ with $\sigma_{k}^{y}=$ $i|g\rangle_{k}\langle r|-i| r\rangle_{k}\langle g|$. Additionally, $\bar{R}_{k}=\sum_{l \in \Lambda_{k}}\left\langle r_{l}\right\rangle$ and we assume that initially no coherences are present. Meanfield will yield qualitatively reasonable predictions unless longrange correlations develop. As we shall show, the transition becomes discontinuous in the quantum regime and is therefore not associated to a divergent correlation length. Our analysis cannot capture the role of spatial dimensionality, but should identify the correct qualitative behavior in sufficiently high dimension; see Ref. [27]. A more detailed analysis could be achieved exploiting cluster mean-field methods [58].

In Fig. 4(a) we show the stationary state density $N_{\text {SS }}$ resulting from Eqs. (5). A striking difference with respect to the classical case is the appearance of an oscillating behavior as a function of the driving parameter $\Omega / \kappa$. From our numerical analysis, it appears that the peaks become sharper as the size of the system is increased, with their positions remaining approximately fixed. This suggests that in the thermodynamic limit a sequence of discontinuous jumps will form at fixed values $\tilde{\Omega}_{j} / \kappa$, $(j=1,2, \ldots)$. Note that a standard numerical analysis of the problem (exact diagonalization, quantum-jump Monte Carlo simulations) is forbiddingly complex, due to the exponential scaling of the state space dimension with the number of atoms.

These jumps feature an intriguing dynamic counterpart: in the quantum case more than one outbreak can occur. As shown in Fig. 4(b), if $\Omega / \kappa$ is too small (first column), no

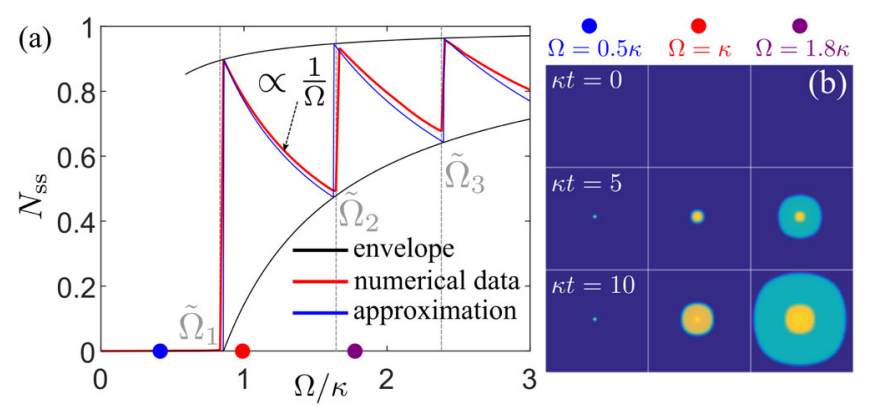

FIG. 4. (a) Stationary state of the mean-field equations (5). We show the numerical solution for the immune density at time $\kappa t=$ 500 starting at $t=0$ from a single infected site located at the center of a $51 \times 51$ lattice. As the driving parameter $\Omega / \kappa$ is increased, a recurrent structure of jumps is seen. (b) Snapshots of the numerical mean-field evolution of the same process for three values of $\Omega$ showing how every jump in $N_{\mathrm{SS}}$ is associated to the appearance of a new outbreak.

outbreak takes place, as in the classical case. For $\Omega \gtrsim \tilde{\Omega}_{1} \approx 0.83 \kappa$, instead, a single outbreak leaves behind an approximately uniform density of immunes (second column). Increasing $\Omega / \kappa$ further, this residual density decreases until, at a second threshold value $\tilde{\Omega}_{2} \approx 1.643 \kappa$, a second outbreak is generated, which causes the final density $N_{\mathrm{SS}}$ to jump to a higher value. Every new jump in $N_{\text {SS }}$ appears to be associated to a new outbreak in the dynamics.

This repeating pattern allows us, by analyzing the first jump, to make predictions on the subsequent ones. To this end, we make two simplifying assumptions compatible with the numerically observed behavior: (i) every outbreak leaves behind a uniform density of immunes and (ii) stems from the center of the lattice. We focus now on a neighborhood of the center after the $(j-1)$ th outbreak, at some time $t_{j}$. By (i) there is an immune density $N_{k}\left(t_{j}\right)=N^{(j)}>0$; by (ii), $R_{k}, G_{k}$, and $\Sigma_{k}$ at time $t_{j}$ correspond to their initial conditions rescaled by $\left(1-N^{(j)}\right)$. Therefore, the facilitation rate is bounded by $\Omega \bar{R}_{k} \leq 4 \Omega\left(1-N^{(j)}\right)$; as a first approximation, the last factor can be reabsorbed by $\Omega \rightarrow \Omega^{(j)}=\Omega\left(1-N^{(j)}\right)$. In other words, the process after $t_{j}$ proceeds like at $t=0$ (no immunes present), but with a modified frequency $\Omega^{(j)}<\Omega$.

Hence, if $\Omega^{(j)}<\tilde{\Omega}_{1}$ the process stops at $N_{\mathrm{SS}}=N^{(j)}$, up to subextensive additions, meaning that $\Omega<\tilde{\Omega}_{j}$. If $\tilde{\Omega}_{1}<\Omega^{(j)}<\tilde{\Omega}_{2}$, instead, a $j$ th outbreak will be produced, but a $(j+1)$ th will not take place, corresponding to $\tilde{\Omega}_{j}<\Omega<\tilde{\Omega}_{j+1}$, and so forth. All the processes sharing the same reference frequency $\Omega^{(j)}$ are thus equivalent to the same reference process occurring in the absence of immunes and their stationary points will lie on a curve $\Omega\left(1-N_{\mathrm{SS}}\right)=$ const. The extremal curves in this set, passing through the top and bottom of the first jump, are displayed in Fig. 4(a) and bound well the data. With 
an ansatz $N_{\mathrm{SS}} \propto 1 / \Omega$ for the decrease between jumps, one can formulate a more detailed prediction out of the same considerations, represented by the blue line (see Ref. [33] for details).

Conclusions. - We have analyzed a simple model for epidemic spreading in an open quantum system, which has been inspired by recent experimental work [15], and investigated its connection with the so-called general epidemic process [29]. In the presence of strong dephasing, the process has a direct relation to the GEP, displaying a continuous transition in the same universality class. In the quantum limit, instead, an intriguingly different physics emerges featuring a sequence of discontinuous jumps. This surprising behavior warrants further theoretical and experimental investigation.

The research leading to these results has received funding from the European Research Council under the European Union's Seventh Framework Programme (FP/2007-2013)/ ERC Grant Agreement No. 335266 (ESCQUMA), the EPSRC Grant No. EP/M014266/1 and the H2020FETPROACT-2014 Grant No. 640378 (RYSQ). R. G. acknowledges the funding received from the European Union's Horizon 2020 research and innovation programme under the Marie Sklodowska-Curie Grant Agreement No. 703683. We are also grateful for access to the University of Nottingham High Performance Computing Facility.

[1] A. Schirotzek, C.-H. Wu, A. Sommer, and M. W. Zwierlein, Phys. Rev. Lett. 102, 230402 (2009).

[2] P. Jurcevic, B. P. Lanyon, P. Hauke, C. Hempel, P. Zoller, R. Blatt, and C. F. Roos, Nature (London) 511, 202 (2014).

[3] R. Islam, R. Ma, P. M. Preiss, M. E. Tai, A. Lukin, M. Rispoli, and M. Greiner, Nature (London) 528, 77 (2015).

[4] T. Fukuhara, S. Hild, J. Zeiher, P. Schauß, I. Bloch, M. Endres, and C. Gross, Phys. Rev. Lett. 115, 035302 (2015).

[5] A. M. Kaufman, M. E. Tai, A. Lukin, M. Rispoli, R. Schittko, P. M. Preiss, and M. Greiner, Science 353, 794 (2016).

[6] J.-y. Choi, S. Hild, J. Zeiher, P. Schauß, A. Rubio-Abadal, T. Yefsah, V. Khemani, D. A. Huse, I. Bloch, and C. Gross, Science 352, 1547 (2016).

[7] M. Schreiber, S. S. Hodgman, P. Bordia, H. P. Lüschen, M. H. Fischer, R. Vosk, E. Altman, U. Schneider, and I. Bloch, Science 349, 842 (2015).

[8] J. Smith, A. Lee, P. Richerme, B. Neyenhuis, P. W. Hess, P. Hauke, M. Heyl, D. A. Huse, and C. Monroe, Nat. Phys. 12, 907 (2016).

[9] M. Saffman, T. G. Walker, and K. Mølmer, Rev. Mod. Phys. 82, 2313 (2010).

[10] R. Löw, H. Weimer, J. Nipper, J. B. Balewski, B. Butscher, H. P. Büchler, and T. Pfau, J. Phys. B 45, 113001 (2012).

[11] P. Schauss, M. Cheneau, M. Endres, T. Fukuhara, S. Hild, A. Omran, T. Pohl, C. Gross, S. Kuhr, and I. Bloch, Nature (London) 491, 87 (2012).

[12] H. Schempp, G. Günter, M. Robert-de Saint-Vincent, C. S. Hofmann, D. Breyel, A. Komnik, D. W. Schönleber,
M. Gärttner, J. Evers, S. Whitlock, and M. Weidemüller, Phys. Rev. Lett. 112, 013002 (2014).

[13] A. Urvoy, F. Ripka, I. Lesanovsky, D. Booth, J. P. Shaffer, T. Pfau, and R. Löw, Phys. Rev. Lett. 114, 203002 (2015).

[14] T. Weber, M. Höning, T. Niederprüm, T. Manthey, O. Thomas, V. Guarrera, M. Fleischhauer, G. Barontini, and H. Ott, Nat. Phys. 11, 157 (2015).

[15] S. Helmrich, A. Arias, and S. Whitlock, arXiv:1605.08609.

[16] M. M. Valado, C. Simonelli, M. D. Hoogerland, I. Lesanovsky, J. P. Garrahan, E. Arimondo, D. Ciampini, and O. Morsch, Phys. Rev. A 93, 040701 (2016).

[17] F. Letscher, O. Thomas, T. Niederprüm, M. Fleischhauer, and H. Ott, Phys. Rev. X 7, 021020 (2017).

[18] E. A. Goldschmidt, T. Boulier, R. Brown, S. Koller, J. T. Young, A. V. Gorshkov, S. L. Rolston, and J. V. Porto, Phys. Rev. Lett. 116, 113001 (2016).

[19] C. Carr, R. Ritter, C. G. Wade, C. S. Adams, and K. J. Weatherill, Phys. Rev. Lett. 111, 113901 (2013).

[20] N. Malossi, M. M. Valado, S. Scotto, P. Huillery, P. Pillet, D. Ciampini, E. Arimondo, and O. Morsch, Phys. Rev. Lett. 113, 023006 (2014).

[21] R. Gutierrez, C. Simonelli, M. Archimi, F. Castellucci, E. Arimondo, D. Ciampini, M. Marcuzzi, I. Lesanovsky, and O. Morsch, arXiv:1611.03288 [Phys. Rev. A (to be published)].

[22] D. Ding, C. Adams, B. Shi, and G. Guo, arXiv:1606.08791.

[23] M. Marcuzzi, J. Minář, D. Barredo, S. de Léséleuc, H. Labuhn, T. Lahaye, A. Browaeys, E. Levi, and I. Lesanovsky, Phys. Rev. Lett. 118, 063606 (2017).

[24] H. Hinrichsen, Adv. Phys. 49, 815 (2000).

[25] G. Ódor, Rev. Mod. Phys. 76, 663 (2004).

[26] M. Marcuzzi, M. Buchhold, S. Diehl, and I. Lesanovsky, Phys. Rev. Lett. 116, 245701 (2016).

[27] M. Buchhold, B. Everest, M. Marcuzzi, I. Lesanovsky, and S. Diehl, Phys. Rev. B 95, 014308 (2017).

[28] M. Henkel, H. Hinrichsen, S. Lübeck, and M. Pleimling, Non-Equilibrium Phase Transitions (Springer, New York, 2008), Vol. 1.

[29] P. Grassberger, Math. Biosci. 63, 157 (1983).

[30] J. L. Cardy, J. Phys. A 16, L709 (1983).

[31] J. L. Cardy and P. Grassberger, J. Phys. A 18, L267 (1985).

[32] H. K. Janssen, Z. Phys. B 58, 311 (1985).

[33] See Supplemental Material at http://link.aps.org/ supplemental/10.1103/PhysRevLett.119.140401 for details, which includes Refs. [34-37]

[34] I. Lesanovsky and J. P. Garrahan, Phys. Rev. Lett. 111, 215305 (2013).

[35] B. Everest, M. Marcuzzi, J. P. Garrahan, and I. Lesanovsky, Phys. Rev. E 94, 052108 (2016).

[36] C. Argolo, Y. Quintino, I. Gleria, and M. Lyra, Physica (Amsterdam) 390A, 1433 (2011).

[37] U. C. Täuber, Critical Dynamics: A Field Theory Approach to Equilibrium and Non-Equilibrium Scaling Behavior (Cambridge University Press, Cambridge, England, 2014).

[38] D. Barredo, H. Labuhn, S. Ravets, T. Lahaye, A. Browaeys, and C. S. Adams, Phys. Rev. Lett. 114, 113002 (2015).

[39] P. Schauß, J. Zeiher, T. Fukuhara, S. Hild, M. Cheneau, T. Macrì, T. Pohl, I. Bloch, and C. Groß, Science 347, 1455 (2015). 
[40] C. Ates, T. Pohl, T. Pattard, and J. M. Rost, Phys. Rev. Lett. 98, 023002 (2007).

[41] T. Amthor, C. Giese, C. S. Hofmann, and M. Weidemüller, Phys. Rev. Lett. 104, 013001 (2010).

[42] M. Gärttner, K. P. Heeg, T. Gasenzer, and J. Evers, Phys. Rev. A 88, 043410 (2013).

[43] D. W. Schönleber, M. Gärttner, and J. Evers, Phys. Rev. A 89, 033421 (2014).

[44] I. Lesanovsky and J. P. Garrahan, Phys. Rev. A 90, 011603 (2014).

[45] N. Thaicharoen, A. Schwarzkopf, and G. Raithel, Phys. Rev. A 92, 040701 (2015).

[46] F. Bouchet and J. Barre, J. Stat. Phys. 118, 1073 (2005).

[47] B. Chatterjee, I. Brouzos, L. Cao, and P. Schmelcher, J. Phys. B 46, 085304 (2013).

[48] U. R. Fischer, A. U. J. Lode, and B. Chatterjee, Phys. Rev. A 91, 063621 (2015).

[49] E. Fasshauer and A. U. J. Lode, Phys. Rev. A 93, 033635 (2016).
[50] H.-P. Breuer and F. Petruccione, The Theory of Open Quantum Systems (Oxford University Press, New York, 2002).

[51] E. Levi, M. Heyl, I. Lesanovsky, and J. P. Garrahan, Phys. Rev. Lett. 116, 237203 (2016).

[52] P. Degenfeld-Schonburg and M. J. Hartmann, Phys. Rev. B 89, 245108 (2014).

[53] M. Marcuzzi, J. Schick, B. Olmos, and I. Lesanovsky, J. Phys. A 47, 482001 (2014).

[54] C. Ates, T. Pohl, T. Pattard, and J. M. Rost, Phys. Rev. A 76, 013413 (2007).

[55] M. Marcuzzi, E. Levi, W. Li, J. P. Garrahan, B. Olmos, and I. Lesanovsky, New J. Phys. 17, 072003 (2015).

[56] H. P. Büchler, E. Demler, M. Lukin, A. Micheli, N. Prokofev, G. Pupillo, and P. Zoller, Phys. Rev. Lett. 98, 060404 (2007).

[57] S. Sevinçli and T. Pohl, New J. Phys. 16, 123036 (2014).

[58] J. Jin, A. Biella, O. Viyuela, L. Mazza, J. Keeling, R. Fazio, and D. Rossini, Phys. Rev. X 6, 031011 (2016). 